\title{
POTENTIAL OF CURATELLA AMARICANA L. AGAINST SARS-COV2: BIOAVAILABILITY, MOLECULAR SIMILARITY AND MOLECULAR DOCKING BETWEEN SECONDARY METABOLITES AND PROTEASE TYPE 3-CHYMOTRYPSIN (3CLPRO)
}

\author{
EMILDO MARCANO ${ }^{1^{*}}$ AND YSBELIA SÁNCHEZ ${ }^{2}$ \\ ${ }^{I}$ Departamento de Química. Laboratorio de Computación de Alto Rendimiento. \\ ${ }^{2}$ Laboratorio de Investigaciones Genéticas. Universidad Nacional Experimental del Táchira (UNET), Venezuela.
}

\begin{abstract}
We report the bioavailability analysis for six secondary metabolites with antiviral, antioxidant and antitumor activity reported, from Curatella amaricana L. Additionally, the molecular similarity analysis of each metabolite is presented and compared with Lopinavir, Ritonavir, Darunavir, Cobicistat and Nelfinavir, which actually are in the third phase for the production of a new vaccine for SARS-Cov2. The mode of interaction through molecular docking between each structure and the zone of action for protease type 3-chymotrypsin (3CLpro) also is presented. The molecular geometry for structures were optimized at semiempirical PM6 level. The bioavailability and molecular docking calculations were performed using the algorithms incorporated in chemoinformatic servers and AutoDock Vina. The results show that the structures studied lead a moderated permeability through the cell membrane, by complying with Lipinski's "rule of 5". Molecular similarity was evaluated by averaging geometric parameters (3D-Shape) and electrostatic potential (ESP). The results show that the most secondary metabolites would have a similar mode of action as the Lopinavir, with average similarity between 0.65 and 0.73 . This last idea is reinforced by the results for molecular docking with the 3 CLpro active site, highlighting the interaction of the molecules studied with the amino acid residues: His-41, Phe-140, Gly-143, Ser-144, Cys-145, His-163, Glu-166 and His-172, with an range interaction-free energy between $-7.2 \mathrm{kcal} / \mathrm{mol}$ and $-9.2 \mathrm{Kcal} / \mathrm{mol}$, highlighting Quercetin 3-O-Alpha-L-rhamnoside with improve affinity energy than Lopinavir.
\end{abstract}

Keywords: SARS-Cov2, secondary metabolites, Curatella amaricana L., protease type 3-chymotrypsin (3CLpro).

\section{INTRODUCTION}

Since the emergence of a new strain of coronavirus called SARS-CoV-2, which causes pneumonia and bronchiolitis, known as covid-19, originating from Wuhan, Hubei, China, a variety of candidate compounds have been reported for the treatment of this virus. According to a recent report, the compounds Lopinavir, Ritonavir, Darunavir, Cobicistat and Nelfinavir are in the third phase for the production of a new vaccine. These compounds have in common their action on protease type 3-chymotrypsin (3CLpro) [1]. 3CLpro together with PLpro (papain-like protease), correspond to the two main proteases in virus replication. These enzymes cleave the two polyproteins PP1A and PP1AB with individual functional components. Due to its role in virus replication, 3CLpro has become a drug target [2].

In order to explore new sources of possible compounds against SARS-CoV-2, we present the bioavailability analysis and molecular docking for six secondary metabolites with antitumor and antiprotease potential, which can be extracted from the plant diversity of our country, Venezuela [3]. Specifically, we have chosen Curatella americana L., a species in the Dilleniaceae family, known in Venezuela as "Chaparro", "Chaparro sabanero" or "Curata". It is a representative pyrophilic tree of the plain and hot land savannas of Venezuela (Amazonas, Aragua, Bolívar, Carabobo, Delta Amacuro, Federal District, Falcón, Lara Miranda, Trujillo and Zulia). This species is recognized by its low and often twisted habit, leathery leaves, thick reddish bark, white flowers and unpleasant smells [4]. With our results, we hope to give a molecular insight to the experimental findings on the use of secondary metabolites of $C$. amaricana $\mathrm{L}$. in the treatment of SARS-CoV-2.

\section{COMPUTATIONAL DETAILS}

\section{Geometric Optimization and Molecular Similarity analysis}

Molecular similarity principle states that molecules with similar structure tend to have similar properties. Indeed, the observation that common substructural fragments lead to similar biological activities, can be quantified from database analysis [5], [6]. The secondary metabolites studied are shown in Figure 1. These compounds are flavonoids derived from Quercetin, whose antiprotease activity is well documented [7]. For the similarity analysis, we used as references the structures of Lopinavir, Ritonavir, Darunavir, Cobicistat and Nelfinavir, which are known for their interaction with 3CLpro.

For all structures, the geometric optimizations were carried out at semiempirical PM6 level using GAMESS software package [8]. The molecular similarity calculations were carried out with ShaEP software package.
ShaEP performs rigid-body superimposition of 3D molecular models, using a matching algorithm [9]. Two characteristic scores were calculated for comparison: 3D shape and electrostatic potential (ESP) [10]. These scores range is from 0 to 1 , in which 0 and 1 correspond to no similarity and the same molecules, respectively.
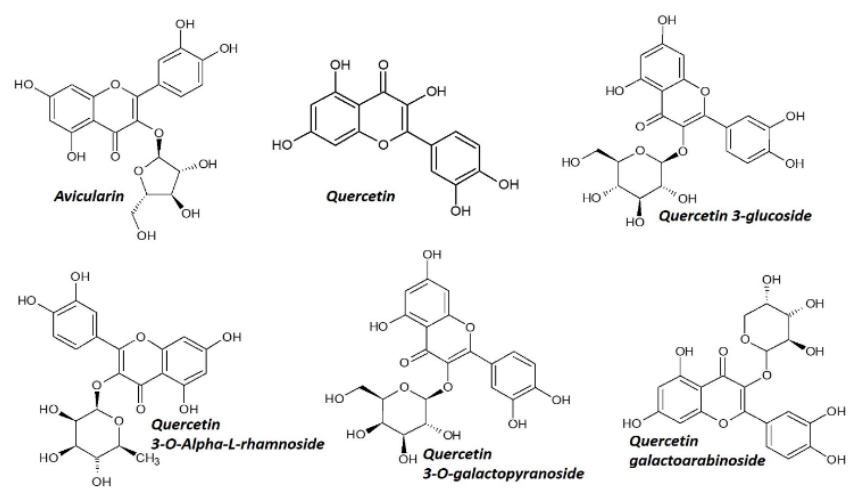

Figure 1. Secondary metabolites of Curatella americana L. ligands studied.

\section{Bioavailability prediction}

Properties of molecules such as bioavailability or membrane permeability have often been connected to simple molecular descriptors such as $\log \mathrm{P}$ (partition coefficient), molecular weight (MW), or counts of hydrogen bond acceptors and donors in molecule [11]. These descriptors are included in the named Lipinski "Rule of Five" [12]. The rule states that most molecules with good membrane permeability have $\log \mathrm{P} \leq 5$, molecular weight $\leq 500$, number of hydrogen bond acceptors $\leq 10$ and number of hydrogen bond donors $\leq 5$. In order to evaluate the bioavailability of the secondary metabolites studied, the Lipinski's parameters were calculated using Molinspiration Cheminformatics software [13].

\section{Preparation of main protease and molecular docking}

Structure of the 3CLpro at $1.82 \AA$ (PDB ID: 2GTB) of resolution was used for studied the interaction with the secondary metabolites of $C$. americana L. [14]. From PDB structure we retained an 3CLpro complex consisting of only A chain (302 residues, from Gly-2 to Cys-300). Hydrogen atoms were added to the model using VEGA ZZ package [15]. Atomic charges were assigned using the 
Gasteiger-Marsili method [16]. Aza-peptide epoxide and others crystal molecules were removed. Secondary metabolites ligands were optimized using semiempirical PM6 level using GAMESS software package. Later, the ligands were prepared for docking experiment defining rotatable bonds using AutoDock Tools version 1.5.6. A grid box size of 50, 60, 50 Á was generated and allocated at the center of the receptor binding site using $\mathrm{x}, \mathrm{y}$ and $\mathrm{z}$ coordinates of 8.876, 4.039 and -1.654. Molecular docking simulations, interactions calculations and visualization of all structures were performed using AutoDock Vina and VEGA ZZ softwares. Co-crystallized ligands were re-docked as validation of the docking protocol.

\section{RESULTS AND DISCUSSION}

\section{Bioavailability (Lipinski parameters)}

Lipinski's rule is widely used to determine molecular properties that are import for drug's pharmacokinetic in vivo. Table 1 contains calculated percentage of absorption (\%ABS), molecular polar surface area (TPSA) and Lipinski parameters of the secondary metabolites investigated.

Table 1. Calculated percentage of absorption (\%ABS), molecular polar surface area (TPSA) and Lipinski parameters of the Curatella americana L. secondary metabolites investigated.

\begin{tabular}{|c|c|c|c|c|c|c|c|c|}
\hline Molecule & $\%$ ABS & Volume & TPSA & Nrot & H acceptors & H donors & $\log P$ & MW \\
\hline Avicularin & 43.4 & 347.36 & 190.28 & 4 & 11 & 7 & 0.80 & 434.35 \\
\hline Quercetin & 63.7 & 240.08 & 131.35 & 1 & 7 & 5 & 1.68 & 302.24 \\
\hline Quercetin 3-O-galactopyranoside & 36.4 & 372.21 & 210.50 & 4 & 12 & 8 & -0.36 & 464.38 \\
\hline Quercetin galactoarabinoside & 43.5 & 347.36 & 190.28 & 3 & 11 & 7 & 0.06 & 434.35 \\
\hline Quercetin 3-glucoside & 36.4 & 372.21 & 210.50 & 4 & 12 & 8 & -0.36 & 464.38 \\
\hline Quercetin 3-O-Alpha-L-rhamnoside & 43.4 & 363.95 & 190.28 & 3 & 11 & 7 & 0.64 & 448.38 \\
\hline
\end{tabular}

Molecular hydrophobicity or lipophilicity is indicated by octanol/water partition coefficient (Log P). Hydrophilic/lipophilic nature of drug molecule affects drug permeability across cell membrane. Log P values of all the secondary metabolites studied were found to be lower than 5, in agreement with Lipinski's rule of five. Quercetin 3-O-galactopyranoside and Quercetin 3-glucoside presents the lowest $\log \mathrm{P}(-0.36)$, indicating that this metabolites are of hydrophilic character, suggesting poor permeability across cell membrane. However, Quercetin and similar falvonoids has been reported for its antiviral activity [17]. In fact, the Quercetin action against SARS-CoV across 3CLpro interaction $\left(\mathrm{IC}_{50}=23.8 \mu \mathrm{M}\right)$ also has been reported [18]. The rest of the metabolites studied have Log P values between 0.64 and 1.68 , indicating greater lipophilicity and therefore better permeability through the cell membrane.

Total polar surface area (TPSA) is closely related to the hydrogen bonding potential of a molecule and is a good predictor of drug transport properties such as intestinal absorption, bioavailability and blood brain barrier penetration. Molecules with a polar surface area of greater than $140 \AA^{2}$ tend to be poor at permeating cell membranes [19]. For molecules to penetrate the blood-brain barrier a PSA less than $90 \AA^{2}$ is usually needed [20]. TPSA for Quercetin lead a value of $131 \AA^{2}$, which in agreement with the limits mentioned above. The rest of the structures studied do not agreement with this range of values. Number of rotatable bonds is a simple topological parameter that measures molecular flexibility and is considered to be a good descriptor of oral bioavailability of drugs. It has been shown that higher oral bioavailability is associated with lower rotatable bond count. Rotational bonds make the compounds flexible, hence easily interact with specific rigid binding area [21]. All the structures studied show low-moderate molecular flexibility due to range of rotatable bonds (1-5). Number of hydrogen bond acceptors ( $\mathrm{O}$ and $\mathrm{N}$ atoms) and number of hydrogen bond donors $(\mathrm{NH}$ and $\mathrm{OH})$ in the tested compounds were found to be not within Lipinski's limit i.e. less than 10 and 5 respectively. The percentages of absorption for title compounds calculated from TPSA ranged between $43 \%$ and $63 \%$, indicated a moderate oral bioavailability. When the BOILED-Egg model [22] was applicated though SwissADME server [23], we found a good permeation blood-brain barrier (BBB) probability for Quercetin

\section{Molecular Similarity}

The aim of this paper is the exploration to the antiviral potential against SARS$\mathrm{CoV}$ of the secondary metabolites of $C$. americana L., through the interaction with 3CLpro. The combination of the literature review and the search for similar structures related to $C$. americana $\mathrm{L}$. secondary metabolites in the PubChem and DrugBank databases, allowed to choose five compounds: Lopinavir, Ritonavir, Darunavir, Cobicistat and Nelfinavir According to a recent report, these compounds are in the third phase for the production of a new vaccine. These compounds have in common their action on 3CLpro [1]. The mechanisms of actions of these drugs are clarified and some molecular targets are validated. and available from the Protein Data Bank. Starting from the knowledge of five molecules identified as antiviral drugs we analyzed the molecular similarity between secondary metabolites of $C$. americana $\mathrm{L}$. Two important properties, 3D-shape and electrostatic potential (ESP) of secondary metabolites were compared to those drugs and the results are shown in Table 2. It is shown that secondary metabolites studied has a high shape similarity with all reference molecules, with average from of 0.63 (Cobicistat) to 0.78 (Nelfinavir). These molecules possess a similar framework of rings, mainly substituted by - $\mathrm{OH}$ groups. It is also shown that the five reference drugs have a high ESP similarity with the most secondary metabolites studied, with average values from 0.67 to 0.73 .

Table 2. 3D-shape and electrostatic potential (ESP) of the Curatella americana L. secondary metabolites investigated

\begin{tabular}{|c|c|c|c|c|c|c|c|c|c|c|}
\hline \multirow{2}{*}{ Molecule/Reference } & \multicolumn{2}{|c|}{ Cobicistat } & \multicolumn{2}{|c|}{ Darunavir } & \multicolumn{2}{|c|}{ Lopinavir } & \multicolumn{2}{|c|}{ Nelfinavir } & \multicolumn{2}{|c|}{ Ritonavir } \\
\hline & shape & $E S P$ & shape & $E S P$ & shape & $E S P$ & shape & $E S P$ & shape & $E S P$ \\
\hline Avicularin & 0.65 & 0.77 & 0.73 & 0.71 & 0.71 & 0.75 & 0.79 & 0.71 & 0.69 & 0.77 \\
\hline Quercetin-3-O-galactopyranoside & 0.67 & 0.74 & 0.73 & 0.64 & 0.74 & 0.75 & 0.81 & 0.75 & 0.72 & 0.75 \\
\hline Quercetin galactoarabinoside & 0.61 & 0.69 & 0.72 & 0.67 & 0.73 & 0.73 & 0.78 & 0.69 & 0.70 & 0.66 \\
\hline
\end{tabular}

When both, 3D-shape and ESP values are averaged, we observed a high similarity of the metabolites studied with Lopinavir. Lopinavir has been reported in experiments of molecular dynamic as a powerful inhibitors of coronavirus protease [24]. In fact, an Arbidol and Liponavir-Ritonavir combination is currently being used in the preclinical testing phase [25]. The molecular similarity of the metabolites studied with Liponavir and the other reference 
molecules, suggest a possible mode of action in the molecular targets of interest for SARS-Cov2.

\section{Molecular Docking}

In the present study, molecular docking was performed to identify the docking score of six structures of the Figure 1 towards of the 3CLpro at 1.82 $\AA$ (PDB ID: 2GTB). Figure 2 and Figure 3 shows the principal interaction mode and affinity energy in the binding site of $3 \mathrm{CL}$ protease for the secondary metabolites studied. His-41, Phe-140, Gly-143, Ser-144, Cys-145, His-163, Glu-166 and His-172 are the main aminoacids in the pocket of 3CLpro, in agreement with the crystallographic structure reported [14].
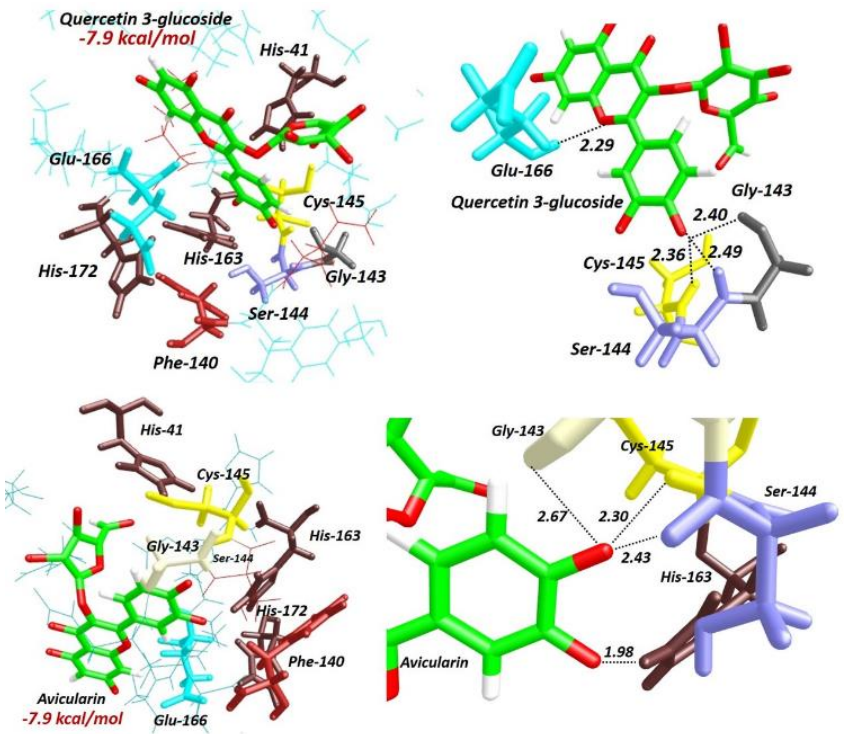

Figure 2. Interaction mode for secondary metabolites ligands in the 3CLpro binding site. Hydrogen bond distances are showed in $\AA$. Affinity energy are showed in $\mathrm{kcal} / \mathrm{mol}$.

The high affinity of drug compounds depends on the type and amount of bonding that occurs with the active site of the protein. Both, Avicularin and Quercetin 3-glucoside molecules have the same affinity energy $(-7.9 \mathrm{kcal} / \mathrm{mol})$. Likewise, both molecules coincide in three of the four hydrogen bonds in the active site of the 3CLpro (Gly-143, Ser-144, Cys-145). However, the stabilization energy due to hydrogen bonding is different, being $-1.21 \mathrm{kcal} / \mathrm{mol}$ for avicularin and $-2.12 \mathrm{kcal} / \mathrm{mol}$ for Quercetin 3-glucoside. This difference can be attributed to the position of the fourth hydrogen bond. For Avicularin this bond is established with His-163 residue and for Quercetin 3-glucoside this bond is formed with Glu-166 residue.

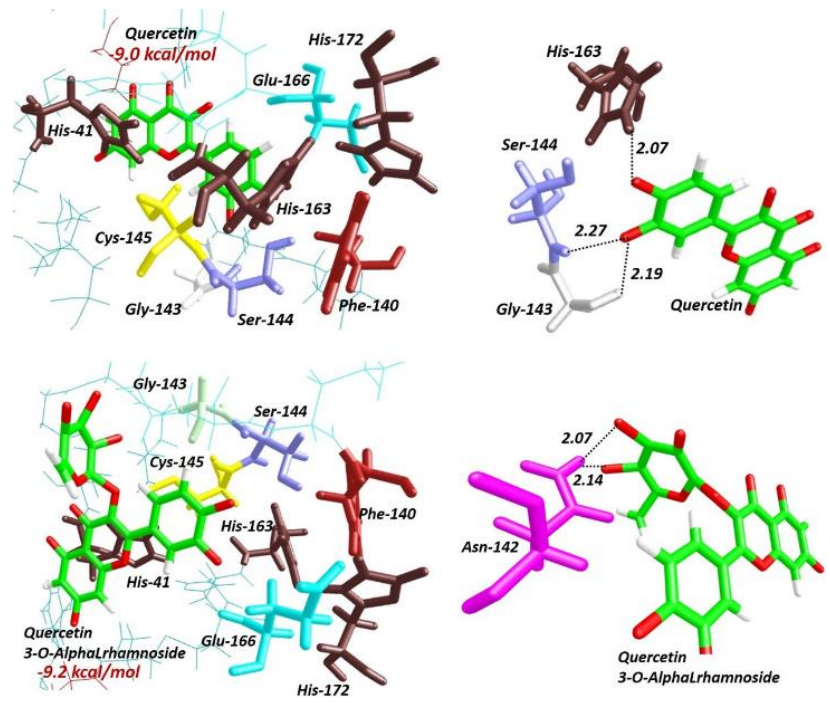

Figure 3. Interaction mode for secondary metabolites ligands in the 3CLpro binding site. Hydrogen bond distances are showed in $\AA$. Affinity energy are showed in $\mathrm{kcal} / \mathrm{mol}$.

Likewise, the stabilization energy by hydrophobic interaction in Quercetin 3glucoside is greater (-6.14 kcal/mol, His-41/Met-49/Cys-145/His-163/Met-165) than Avicularin (-5.44 kcal/mol, His-41/Met-49/Cys-145/His-163) due to additional interaction with Met-165.

Quercetin and Quercetin 3-O-Alpha-L-rhamnoside shows the higher affinity energy values, $-9.0 \mathrm{kcal} / \mathrm{mol}$ and $-9.2 \mathrm{kcal} / \mathrm{mol}$, respectively (Figure 3 ). Both molecules interacting in the active site of the 3CLpro with His-41, Phe-140, Gly143, Ser-144, Cys-145, His-163, Glu-166 and His-172. The stabilization energy due to hydrogen bonding is different, being $-1.79 \mathrm{kcal} / \mathrm{mol}$ for Quercetin 3-OAlpha-L-rhamnoside (Asn-142) and $-2.68 \mathrm{kcal} / \mathrm{mol}$ for Quercetin (Gly-143/Ser144/His-163). Regarding hydrophobic interactions, the stabilization energy values are similar, being $-3.25 \mathrm{kcal} / \mathrm{mol}$ for Quercetin 3-O-Alpha-L-rhamnoside and $-3.38 \mathrm{kcal} / \mathrm{mol}$ for Quercetin. As mentioned earlier in this article, the potential of flavonoids as possible agents against SARS-Cov2 has been widely reported [26]. In order to compare with the reference compounds and their action with 3CLpro, the Table 3 show the affinity energy for the all the compounds.

Table 3. Energy affinity (kcal/mol) for de secondary metabolites studied and reference compounds.

\begin{tabular}{|l|c|}
\hline Molecule & Affinity energy \\
\hline Avicularin & $-7,9$ \\
\hline Quercetin & $-9,0$ \\
\hline Quercetin-3-O-galactopyranoside & $-7,8$ \\
\hline Quercetin galactoarabinoside & $-8,7$ \\
\hline Quercetin 3-glucoside & $-7,9$ \\
\hline Quercetin 3-O-Alpha-L-rhamnoside & $-9,2$ \\
\hline Cobicictat & $-8,1$ \\
\hline Darunavir & $-7,8$ \\
\hline Lopinavir & $-8,5$ \\
\hline Nelfinavir & $-9,9$ \\
\hline Ritonavir & $-7,0$ \\
\hline
\end{tabular}

Quercetin and its derivate Quercetin 3-O-Alpha-L-rhamnoside shows the bes energy affinity, in accordance with the energy affinity Nelfinavir. Has been reported that the HIV-1 protease inhibitor, nelfinavir, strongly inhibite replication of the SARS coronavirus (SARS-CoV). Nelfinavir inhibite the cytopathic effect induced by SARS-CoV infection and the expression of viral antigens is much lower in infected cells treated with nelfinavir than in untreated infected cells [27]. On the other hand, Quercetin galactoarabinoside values for energy affinity are comparable with the energy affinity for both, Cobicistat and Lopinavir. According to a recent report, Lopinavir and Cobicistat are in the third phase for the production of a new vaccine against SARS-CoV2 infection [28]; [1]. According to our results, the significant molecular similarity and bioavailability found in the secondary metabolites derived from quercetin in $C$. americana $\mathrm{L}$., this specie is projected as a source of extraction for new compounds in the fight against SARS-Cov2.

\section{CONCLUSIONS}

Curatella americana L. is a specie found in Latin America with a strong presence in the plain and hot land savannas of Venezuelan. Theoretical and experimental reports on the activity against SARS-Cov2 of flavonoids found in other plant species around the world, suggest $C$. americana as a possible source for these compounds. In this framework of ideas, we have determined the molecular similarity and bioavailability of the secondary metabolites derived from quercetin found in this specie. Our results show significant similarity with some antiviral reference compounds such as Lopinavir and Nelfinavir, which have currently been used against SARS-Cov2. The bioavailability of the studied 
compounds is in moderate agreement with the Lipinski "Rule of Five". On the other hand, molecular doking at the 3CLpro active binding site show affinity

\section{ACKNOWLEDGMENTS}

The authors acknowledge and thank they're to the Universidad Nacional Experimental del Táchira-Venezuela and its Decanato de Investigación for all supporting this research.

\section{DECLARATION OF INTERESTS}

The authors declare that they have no known competing financial interests or personal relationships that could have appeared to influence the work reported in this paper.

\section{REFERENCES}

1. G. L. Clercq and E. De, "Therapeutic options for the 2019 novel coronavirus (2019-nCoV)," Nat. Rev. Drug Discov, vol. 19, pp. 149-150, 2020. DOI: https://10.1038/d41573-020-00016-0.

2. Yu Wai Chen, Chin-Pang Bennu Yiu and Kwok-Yin Wong, "Prediction of the SARS-CoV-2 (2019-nCoV) 3C-like protease (3CLpro) structure: virtual screening reveals velpatasvir, ledipasvir, and other drug repurposing candidates," F1000 Research, vol. 9, p. 129, 2020. DOI: https://10.12688/f1000research.22457.2.

3. P. G. Taylor, I. M. Cesari, M. Arsenak, D. Ballen, M. J. Abad, A. Fernández, B. Milano, M.-C. Ruiz and B. W. \&. F. Michelangeli, "Evaluation of Venezuelan Medicinal Plant Extracts for Antitumor and Antiprotease Activities," Pharm. Biol., vol. 44, pp. 349-362, 2006. DOI: https://doi.org/10.1080/13880200600748119.

4. R. Duno de Stefano, G. Aymard and O. Huber, Flora Vascular de los Llanos de Venezuela, Caracas . Fundación Empresas Polar - FUNDENA - FIBV, 2007, p. 738 páginas.

5. A. Bender and R. C. Glen, "Molecular similarity: a key technique in molecular informatics," Org. Biomol. Chem., vol. 2, pp. 3204-3218, 2004. DOI: https://doi.org/10.1039/B409813G.

6. H. Eckert and J. Bajorath, "Molecular similarity analysis in virtual screening: foundations, limitations and novel approaches," Drug Discov Today, vol. 12, pp. 225-233, 2007. DOI: https://doi.org/10.1016/j.drudis.2007.01.011.

7. S. Jo, S. Kim and D. H. S. \&. M.-S. Kim, "Inhibition of SARS-CoV 3CL protease by flavonoids," J. Enzyme Inhib., vol. 35, pp. 145-151, 2020. DOI: https://doi.org/10.1080/14756366.2019.1690480.

8. M. W. Schmidt, K. K. Baldridge, J. A. Boatz, S. T. Elbert, M. S. Gordon, J. H. Jensen, S. Koseki, N. Matsunaga, K. A. Nguyen, S. Su, T. L. Windus, M. Dupuis and J. A. Montgomery J, "General Atomic and Molecular Electronic Structure System," Comput. Chem., vol. 14, pp. 1347-1363, 1993. DOI: https://doi.org/10.1002/jcc.540141112.

9. M. J. Vainio, J. S. Puranen and M. S. Johnson, "ShaEP: molecular overlay based on shape and electrostatic potential.," J Chem Inf Model., vol. 49, pp. 492-502., 2009. DOI: https://doi.org/10.1021/ci800315d.

10. W.-H. Shin, Z. Xiaolei, M. G. Bures and D. Kihara, "Three-Dimensional Compound Comparison Methods and Their Application in Drug Discovery," Molecules, vol. 20, pp. 12841-12862, 2015. DOI: https://doi.org/10.3390/molecules200712841.

11. Muegge, "Selection Criteria for Drug-Like Compounds," Med.1 Res. Rev, vol. 23, pp. 302-321, 2003. DOI: https://doi.org/10.1002/med.10041.

12. C. A. Lipinski, F. Lombardo, B. W. Dominy and P. J. Feeney, "Experimental and computational approaches to estimate solubility and permeability in drug discovery and development settings," Adv. Drug Deliv. Rev, vol. 15, pp. 325, 1997. DOI: https://doi.org/10.1016/S0169-409X(96)00423-1

13. Molinspiration , "Cheminformatics on the Web," 1986. [Online]. Available: http://www.molinspiration.com/.

14. T.-W. Lee, M. M. Cherney, J. Liu, K. E. James, J. C. Powers, L. D. Eltis and M. N. James, "Crystal Structures Reveal an Induced-fit Binding of a Substrate-like Aza-peptide Epoxide to SARS Coronavirus Main Peptidase," J. Mol. Biol., vol. 366, pp. 916-932, 2007. DOI: https://doi.org/10.1016/j.jmb.2006.11.078.

15. Pedretti, L. Villa and G. Vistoli, "VEGAZZ: an open platform to develop chemo-bio-informatics applications, using plug-in architecture and script programming," J Comput Aided Mol Des, vol. 18, pp. 167-173 , 2004.DOI: https://doi.org/10.1023/B:JCAM.0000035186.90683.f2.

16. J. Gasteiger and M. Marsili, "Iterative partial equalization of orbital electronegativity - a rapid access to atomic charges," Tetrahedron, vol. 36, pp. 3219-3228, 1980. DOI: https://doi.org/10.1016/0040-4020(80)80168-2 energies of up to $-9.2 \mathrm{kcal} / \mathrm{mol}$, which coincides with the values found in various molecular targets of SARS-Cov2.

17. H. Zakaryan, E. Arabyan, A. Oo and K. Zandi, "Flavonoids: promising natural compounds against viral infections.," Arch. Virol., vol. 162, p. 2539 2551, 2017. DOI: https://doi.org/10.1007/s00705-017-3417-y.

18. Y. B. Ryu, H. J. Jeong, J. H. Kim, Y. M. Kim, J.-Y. Park, D. Kim, T. T. H. Naguyen, S.-J. Park, J. S. Chang, K. H. Park, M.-C. Rho and W. S. Lee, "Biflavonoids from Torreya nucifera displaying SARS-CoV 3CLpro inhibition," Bioorg. Med. Chem., vol. 18, pp. 7940-7947, 2010. DOI: https://doi.org/10.1016/j.bmc.2010.09.035.

19. H. Pajouhesh and G. R. Lenz, "Medicinal Chemical Properties of Successful Central Nervous System Drugs," NeuroRx, vol. 2, p. 541-553, 2005. DOI: https://doi.org/10.1602/neurorx.2.4.541.

20. S. A. Hitchcock and L. D. Pennington, "Structure-Brain Exposure Relationships," J. Med. Chem., vol. 49, pp. 7559-7583, 2006. DOI: https://doi.org/10.1021/jm060642i

21. D. Veber, S. Johnson, H. Cheng, B. Smith, K. Ward and K. Kopple, " Molecular properties that influence the oral bioavailability of drug candidates," J Med Chem, vol. 45, pp. 2615-2623, 2002. DOI: https://doi.org/10.1021/jm020017n.

22. Daina and Z. Vincent, "A BOILED-Egg To Predict Gastrointestinal Absorption and Brain Penetration of Small Molecules," ChemMedChem, vol. 11, p. 1117-1121, 2016. DOI: https://doi.org/10.1002/cmdc.201600182

23. A. Daina, O. Michielin and V. Zoete, "SwissADME: a free web tool to evaluate pharmacokinetics, drug-likeness and medicinal chemistry friendliness of small molecules.," Sci. Rep., vol. 7, p. 42717, 2017. DOI: https://doi.org/10.1038/srep42717.

24.D. M, Taleb-Gassabi and D. M, "Lopinavir; A Potent Drug against Coronavirus Infection: Insight from Molecular Docking Study," Arch. Clin. Infect. Dis., vol. 12, p. e13823, 2017. DOI: 10.5812/archcid.13823.

25. L. Deng, C. Li, Q. Zeng, X. Liu, X. Li, H. Zhang, Z. Hong and J. Xia, "Arbidol combined with LPV/r versus LPV/r alone against Corona Virus Disease 2019: A retrospective cohort study," J. Infect., vol. Article in press, 2020. DOI: https://doi.org/10.1016/j.jinf.2020.03.002.

26. J. Cui, X. Liu and L. M. Chow., "Flavonoids as P-gp Inhibitors: A Systematic Review of SARs," Curr. Med. Chem. vol. 26, pp. 4799-4831, 2019. DOI: 10.2174/0929867325666181001115225.

27. N. Yamamoto, R. Yang, Y. Yoshinaka, S. Amari, T. Nakano, J. Cinatl, H. Rabenau, H. W. Doerr, G. Hunsmann, A. Otaka, H. Tamamura, N. Fujii and N. Yamamoto, "HIV protease inhibitor nelfinavir inhibits replication of SARS-associated coronavirus," Biochem. Biophys. Res. Commun., vol. 318, pp. 719-725, 2004. DOI: https://doi.org/10.1016/j.bbrc.2004.04.083.

28. T. Sheahan, A. Sims and S. Leist, "Comparative therapeutic efficacy of remdesivir and combination lopinavir, ritonavir, and interferon beta against MERS-CoV," Nat. Commun., vol. 11, p. 222, 2020. DOI: https://doi.org/10.1038/s41467-019-13940-6.

29. S. K. Bhal, "LogP-Making Sense of the Value," 2012. [Online]. Available: http://www.acdlabs.com/logp. 